ENCYCLOPÉDIE Encyclopédie berbère

BERBERE $\mathbf{3 3 |} \mathbf{2 0 1 2}$

33| N - Nektiberes

\title{
Nefzaoua : Antiquité
}

Pol Trousset

\section{(2) OpenEdition}

Journals

Édition électronique

URL : https://journals.openedition.org/encyclopedieberbere/2703

DOI : 10.4000/encyclopedieberbere. 2703

ISSN : 2262-7197

\section{Éditeur}

Peeters Publishers

\section{Édition imprimée}

Date de publication : 4 octobre 2012

Pagination : 5382-5389

ISBN : 978-90-429-2640-0

ISSN : 1015-7344

\section{Référence électronique}

Pol Trousset, « Nefzaoua : Antiquité », Encyclopédie berbère [En ligne], 33 | 2012, document N32, mis en ligne le 23 novembre 2020, consulté le 17 février 2022. URL : http://journals.openedition.org/ encyclopedieberbere/2703 ; DOI : https://doi.org/10.4000/encyclopedieberbere.2703

Ce document a été généré automatiquement le 17 février 2022.

(c) Tous droits réservés 


\title{
Nefzaoua : Antiquité
}

\author{
Pol Trousset
}

1 Nefzaoua est un nom qui désigne à la fois un groupe de peuples berbères partiellement arabisés à partir des $\mathrm{XI}^{\mathrm{e}-\mathrm{XII}}{ }^{\mathrm{e}}$ siècles et la région qu'ils occupaient depuis l'Antiquité. Ce territoire est situé entre le Chott el-Djérid à l'ouest, le Chott el-Fedjedj au nord, le plateau du Dahar à l'est et les dunes du Grand Erg au sud. Il est traversé d'est en ouest par l'arc montagneux du Djebel Tebaga jusqu'à la presqu'île étroite formée par les deux Chotts où se trouve le Bled Faraoun. Cet enclavement entre les grandes sebkhas, les montagnes présahariennes et les sables du désert, peut expliquer que ce pays et ses peuples aient vécu longtemps en marge des autres régions de l'Afrique du Nord. À cet isolement très relatif, nous le verrons, s'ajoute la dispersion des oasis beaucoup moins concentrées que dans le Bled el-Djérid voisin. On compte aujourd'hui au Nefzaoua plus de 200 sources et plus de 30 agglomérations, presque toutes modestes (Despois \& Raynal, p. 429; Sethom \& Kassab, p. 130-131). Une telle configuration géographique peut expliquer la survivance plus marquée qu'ailleurs du peuplement tribal berbère, mais elle a aussi, paradoxalement, semblé donner un fondement à l'hypothèse d'une origine très lointaine de ces populations, les Louata ou Nefzaoua, qui seraient venus d'Égypte par une longue migration, pour trouver refuge dans ce finistère du pays des Chotts. Ainsi s'expliquerait le nom de Faraoun déjà cité dans la région. On s'est référé souvent à ce sujet à Ibn Khaldoûn pour qui l'origine du nom de Nefzaoua viendrait de celui de Nefzao, ancêtre d'une des branches berbères du groupe des Botr venus de l'est (Moreau, p. 5, 58). Mais comme l'avait récemment démontré Yves Modéran (p. 157-162), le mythe de l'origine orientale des Louata et autres Botr aurait sa source dans un texte du même Ibn Khaldoûn qui se rattache à une légende sur l'origine des peuples à replacer dans les temps bibliques à l'est du Nil: "Les Berbères sont les enfants de Canaan, fils de Cham, fils de Noë ; leur aïeul se nommait Mazigh... ».

2 En fait, le pays des Nefzaouas, malgré sa situation en cul-de-sac qui en aurait fait une sorte de refuge pour leurs ancêtres Louatas et Nasamons plus ou moins mythiques, est bien aussi un espace de circulation comme le met en évidence le réseau des voies romaines établies plus tard dans ce qui deviendra la zone-frontière du limes : un axe routier essentiel est celui qui à partir de Gabès (Tacape), après avoir longé le Chott elFedjedj et le Djebel Tebaga, rejoint le centre de Telmine (la civitas Nybgeniorum ou Turris 
Tamalleni). Une autre voie partie de Gafsa (Capsa) traversait le Chott el-Fedjedj en direction de cette cité des Nyhgéniens. Un passage plus saisonnier encore sans doute, traversait le Chott el-Djerid en direction des oasis de la presqu'île du Nefzaoua à partir des Oudiane, ce que prouve l'inscription du rocher des Sebaa Argoud et sa dédicace à Mercure, le dieu des passeurs. Ce passage restera utilisé, malgré ses dangers, pendant l'époque arabe d'après les témoignages d'El-Bekrî et de Tijanî; il sera même fréquenté par les pèlerins se rendant du Maroc à la Mecque jusqu'au XIX ${ }^{e}$ siècle. Un autre itinéraire contournait le Chott el-Djerid par le sud entre Nefta et la région de Douz. Il était sans doute utilisé quand les autres passages n'étaient pas praticables. De ces aléas vient sans doute la réputation de passeurs des Arzuges, un des peuples de la région qui donneront leur nom dans l'Antiquité tardive à l'ensemble de la région du Bled el-Djérid et du Nefzaoua (l'Arzugis)

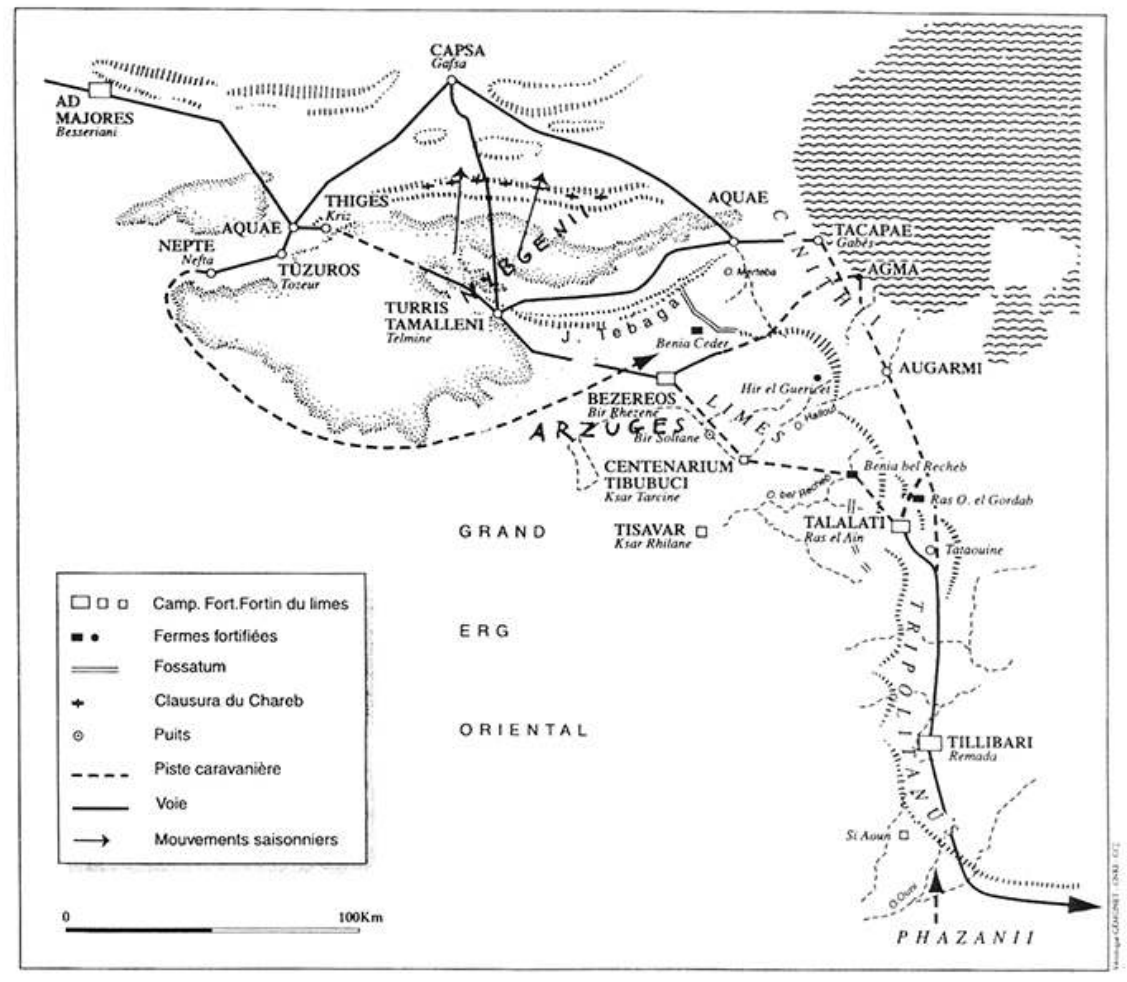

Fig. 1. CARTE du NEFZAOUA DANS SON ENVIRONNEMENT gÉograPhiQUE.

En s'interposant entre Steppe et Sahara, ce pays des Chotts ne constitue donc nullement un isolat; il commande bien des axes de mobilité orientés vers l'isthme de Gabès et la Petite Syrte à l'Est, vers Capsa et les Hautes Steppes au nord, vers le piedmont saharien et les Zibans à l'Ouest. Par les vallées des oueds Hallouf et Bel Recheb ou de leurs affluents descendant du Djebel Demmer, des itinéraires jalonnés de points d'eau fréquentés dès l'Antiquité conduisaient vers des passages qui débouchaient, à travers la montagne, dans la plaine de la Djeffara. Des dispositifs de contrôle romains - murs et fossés, avant-postes et tours de guet - y avaient été mis en place aux environs du camp de Talalati (Tlalet). Enfin, plus au sud encore, en s'engageant par Ksar Rhilane, dans les premières dunes du Grand Erg, des pistes plus lointaines conduisaient vers Remada (Tillibari), Ghadamès (Cidamus) et le Fezzan. Parmi les lieux de passage les plus significatifs à toute époque - de la période romaine à la deuxième guerre mondiale - parce qu'il permettait de contourner par l'intérieur la plaine côtière de l'isthme de Gabès, se distingue le seuil stratégique situé aux confins du 
Nefzaoua, entre le Djebel Tebaga et l'extrémité du massif des Matmata* (le Djebel Melab). À l'époque romaine il avait été doté d'une grandiose défense linéaire permettant de contrôler les passages : on peut y voir une muraille doublée d'un fossé de $17 \mathrm{~km}$ de longueur et l'emplacement d'un poste de contrôle. Pendant la deuxième guerre mondiale les forces alliées - dont la colonne Leclerc venue de Ksar Rhilane avaient réussi à forcer ce passage en évitant ainsi de se heurter aux défenses de la ligne Mareth.

4 La raison la plus fondamentale de l'ouverture de la région est liée au genre de vie de ses populations qui, de tout temps, s'est partagé entre les activités de cultivateurs sédentaires cantonnées dans les oasis et les déplacements saisonniers nomades ou semi-nomades dont le rayon d'action débordait largement du cadre géographique du Nefzaoua stricto sensu. On retrouve dans les groupes tribaux actuels de la région - les Ghrib, Ouled Sidi Abid, Ouled Bou Yaya, Ouled Yacoub, Sabria, Adhara et Marâzig - un schéma de mobilité dont on peut croire qu'il pérennise des axes de transhumance remontant à l'Antiquité. Ainsi pour les Marâzig de la région de Douz, dont l'aire de transhumance s'étendait largement, sur le revers montagneux du Dahar et surtout dans le grand Sud jusqu'aux environs de Ghadamès (Moreau, p. 83, 122). Leur nom viendrait d'un certain Sidi Marzig venu de Tripolitaine et qui aurait réuni sous son autorité maraboutique des Berbères sahariens nomades et quelques sédentaires de la région de Douz pendant les invasions hilaliennes. Il serait donc peut-être risqué de proposer comme l'avait fait Courtois (p.93), une filiation possible entre ce nom et celui des Arzuges de l'Antiquité. En revanche une continuité plus grande se laisse deviner plus au nord dans les oasis de la presqu'île du Nefzaoua en particulier autour de Telmine - le pays des Nybgéniens de l'Antiquité - dont la population est plus sédentaire mais où des groupes de semi-nomades semblent avoir, depuis les temps les plus anciens, fréquenté des aires transhumance ou de labour situées au nord du Chott el-Fedjedj, dans les plaines et montagnes du Chareb et dans le Bled Segui où ils étaient en symbiose ou en concurrence avec d'autres peuples et cités gétules* de cette partie de la Haute Steppe (Fig. 1).

5 C'est précisément dans ces régions septentrionales du Nefzaoua, à l'interface du prédésert et de la steppe qu'on est le mieux documenté sur les étapes de la pénétration romaine avant la mise en place plus au sud des installations militaires du limes Tripolitanus. L'événement décisif fut sans aucun doute la révolte de Tacfarinas* entre 14 et 27 ap. J.-C., dans laquelle les peuples du Nefzaoua ont pu être impliqués comme l'avaient été les Cinithii dans une région voisine le long de la Petite Syrte. Pour prévenir le retour de telles tribulations, force était pour le commandement militaire romain de prendre, par étapes, le contrôle du glacis présaharien où s'étaient nouées, le long des axes de déplacements des nomades et semi-nomades, de vastes coalitions tribales pouvant réunir les Garamantes* du grand Sud et les Musulames* de la steppe au nordouest.

Dès le règne de Tibère, le prélude à la pénétration romaine sur ce glacis est l'opération d'arpentage lancée à partir du camp de la III ${ }^{\mathrm{e}}$ Légion d'Ammaedara sous le Proconsul d'Afrique C. Vibius Marsus en 29-30 ap. J.-C. et dont les bornages ont été découverts sur les deux rives du Chott el-Fedjedj ainsi que dans le Bled Segui. Cette centuriation servira de base aux délimitations effectuées entre les territoires de tribus et de cités au cours de la période suivante, sous le règne de Trajan tout particulièrement. C'est ainsi que le nom des $\mathrm{Nyb}$ (genii) figure sur des bornes marquant la limite entre ce peuple et la 
cité de Tacapae ainsi que sur des milliaires datés du même règne, en 105, au sud du Djebel el-Asker, sur la route entre Capsa et la civitas Nybgeniorum. On peut penser comme le suggère $M$. Rachet (p.163), que les Nybgéniens durent céder une partie de leur territoire aux deux cités déjà romanisées. À tout le moins leurs déplacements et surtout celui d'autres nomades venus de plus loin, furent-ils soumis à un contrôle rigoureux comme en témoignent les puissantes murailles - dont celle de Bir Oum Ali érigées à une époque non encore connue mais sans doute plus tardive, au $\mathrm{III}^{\mathrm{e}}$ siècle peut-être, dans les chaînons du Chareb qui correspondent à la limite entre les territoires de Capsa et des Nybgenii. Il en est de même pour la muraille du Tebaga érigée à l'entrée de l'Aradh où se trouve Tacapae.

7 En fait, le cas de la civitas Nybgeniorum qui obtiendra le statut de municipe dès le règne d'Hadrien en 128 est une bonne illustration du processus de mise en tutelle, mais aussi de promotion des gentes et civitates, lesquelles vont rentrer peu à peu dans les cadres civiques de la romanité selon des modalités semblables à celles des autres régions de l'Africa. Situé à un carrefour de pistes remarquable du Nefzaoua dans le groupe d'oasis de Telmine et de Mansourah, ce centre urbain deviendra par la suite un des points forts du limes Tripolitanus mentionné par l'Itinéraire Antonin sous le nom de Turris Tamalleni où se reconnaît le toponyme actuel de Telmine dont le sens est peut-être à rapprocher du terme berbère temellé = «blanche/blancheur ». Des vestiges antiques y avaient été reconnus depuis longtemps par R. Donau et R. Cagnat : à Torra, quartier de Mansourah, près de la source de l'Aïn Rherig (Fig. 2) se remarquent un barrage et les restes d'un ancien bordj réutilisant des matériaux romains et les fragments d'une dédicace impériale d'époque sévérienne y avaient été recueillis (CIL VIII, 23160-23164). On a tout lieu de penser que le commandement militaire romain avait installé en ce lieu un poste qui pourrait avoir été plus tard le siège du praepositus limitis Tamallensis, à la tête de ce secteur du limes d'après la Notitia Dignitatum.

Un autre poste militaire romain d'importance se trouvait plus à l'Est, aux environ de Bir Rhezen près du puits de Mohammed ben Aïssa, sur une piste allant du Nefzaoua à Tamezret* près des Matmata. Il s'agit du camp de Bezereos daté du règne de Commode et mentionné à la fois par l'Itinéraire Antonin sur la route frontière du limes Tripolitanus et au $\mathrm{V}^{\mathrm{e}}$ siècle par la Notitia Dignitatum où le limes Bizerent(anus) est un sous-secteur de la frontière de Tripolitaine entre le limes Tamallensis et le limes Talalatensis (près de Tataouine). On peut en conclure à la fois une continuité de dessein dans la pénétration de l'armée romaine sur ces confins sahariens et le maintien de sa présence jusqu'au Bas Empire. Deux avant-postes complétaient ce dispositif pour contrôler grâce à des détachements de cavalerie les abords sahariens du Nefzaoua; il s'agit du centenarium Tibubuci (Ksar Tarcine) situé sur l'Oued Hallouf qui conduit au Dahar et, plus au sud encore, du castellum de Tisavar (Ksar Rhilane) : adossé aux premières dunes du Grand Erg, à mi-distance du Nefzaoua et du camp de Remada, il surveillait l'itinéraire le plus court entre Ghadamès et le Djérid. 


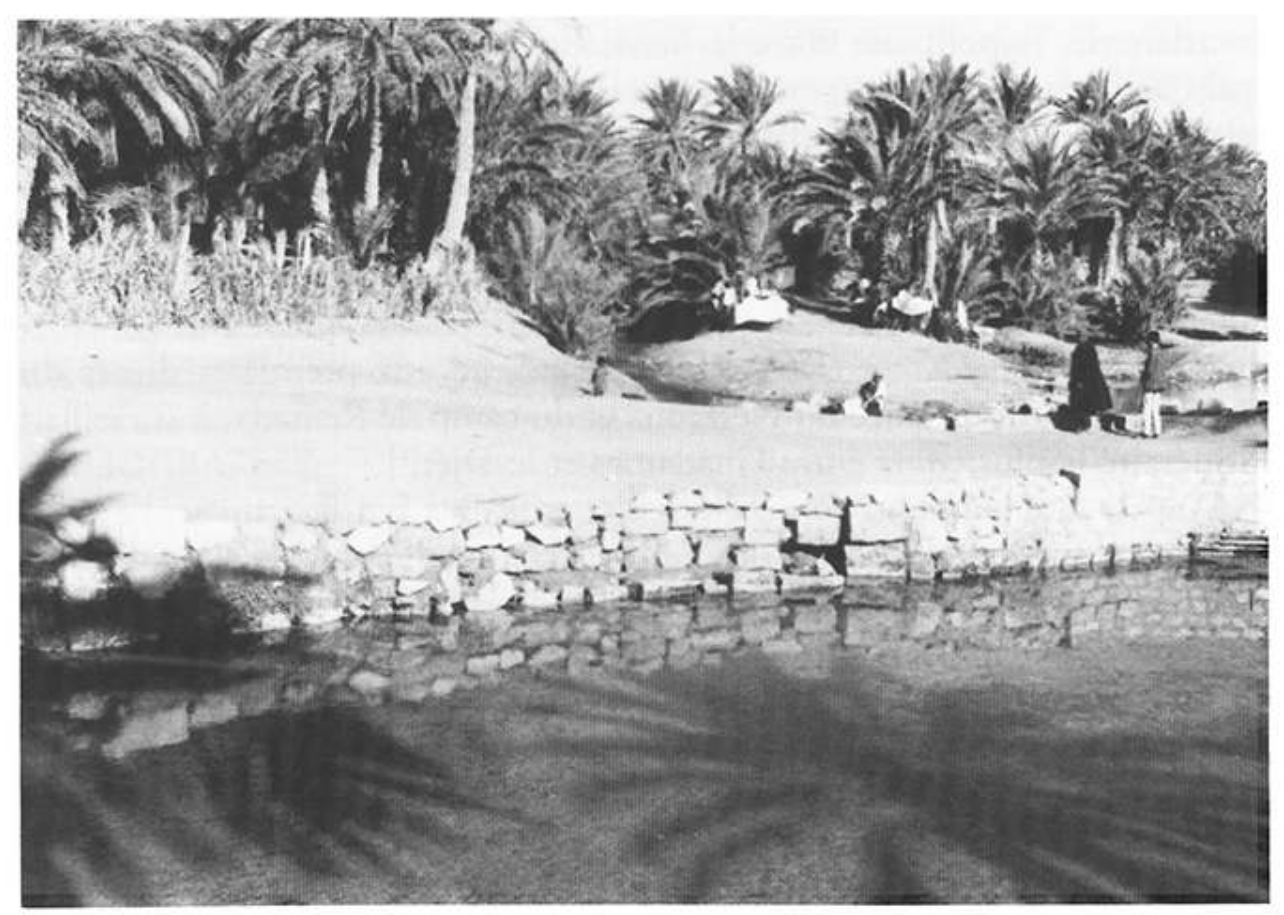

Fig. 2. SOURCE ET BARRAge de MANSOURAH PRÈs d'Aïn REZIg DANS LE NEFZAOUA

Cliché P. Trousset.

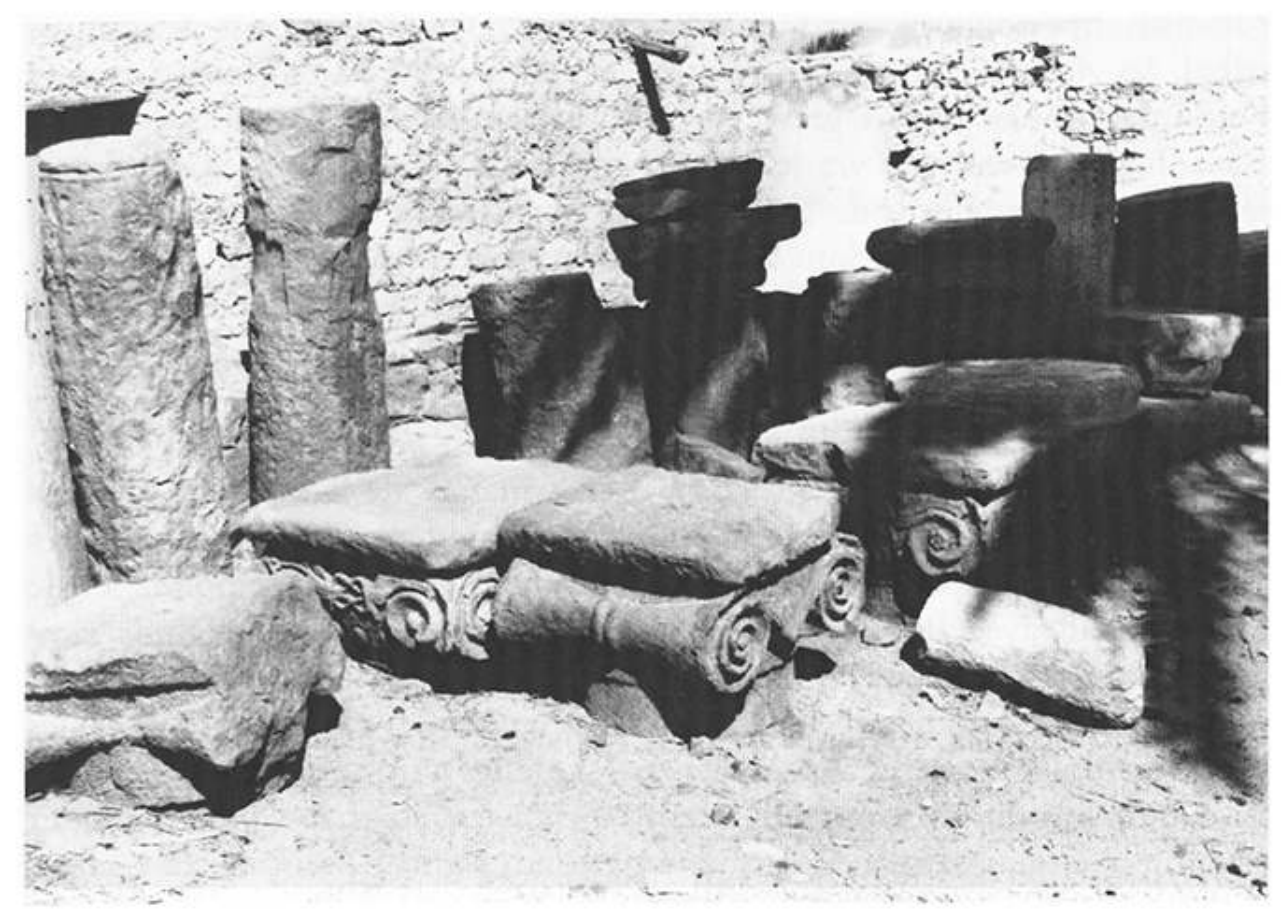

Fig. 3. eléments d'ARchitecture dANS LA CoUr de L'ANCIENNe MOSQuÉE de telmine.

D'après la documentation ecclésiastique du Bas Empire, un seul évêché est connu sur toute l'étendue du Nefzaoua actuel, il s'agit du siège de Turretamalliensis ou Turretamallumensis qui correspond bien au site de Telmine où des chapiteaux tardifs pouvant provenir d'un édifice chrétien ont été effectivement signalés dans la cour de la mosquée (Fig. 3). Les noms de trois évêques y sont connus pour avoir participé aux conciles de Carthage, le premier (Gaudentius) en 348, les deux autres en 411, Sabratius 
évêque catholique ayant pour rival le donatiste Iurata (Mandouze, p. 1020). Sous le nom d'Arzugis qui désigne alors le Nefzaoua avec les évêchés voisins sur l'autre rive du Djérid, le pays des Chotts constituait alors comme l'a démontré Y. Modéran (p. 368), une subdivision de la province ecclésiastique de Byzacène. Ce nom même qui finit par désigner chez les auteurs anciens comme Orose (trad. Y. Janvier, p. 53) un vaste ensemble territorial mal défini entre Tripolitaine et Byzacène et tout au long de la frontière romaine, tire son origine d'un ethnonyme qui s'appliquait à un groupe tribal, les Arzuges dont le territoire avait été délimité sous Trajan, comme l'indique le nom Arzosei gravé sur une borne découverte aux environs de Bir Soltane. Selon toute vraisemblance son territoire de rattachement devait se trouver aux environs de Douz dans la partie sud du Nefzaoua. À la différence des Nybgéniens dont la sédentarisation fut rapide après la délimitation de leur territoire, ce que prouve la densité des établissements ruraux dans la partie nord du Nefzaoua, les Arzuges pourraient avoir conservé à la fois leur structure tribale et leur mobilité traditionnelles. Ceci explique leur réputation de passeurs ou de guides attachée à leur nom (Desanges, EB, VI, p. 950-952). De plus, leur situation sur les marges de la zone du limes les mettait en contact avec des populations non chrétiennes restées extérieures à l'Empire. Sur ce point, le document souvent cité est la correspondance de St. Augustin avec Publicola propriétaire de terres dans ces régions éloignées; non résident, il s'en remettait pour l'exploitation de ses biens à des conductores. et s'inquiétait de la souillure religieuse qui pouvait résulter des serments idolâtres prêtés auprès de ceux-ci ou des autorités militaires locales par une main-d'œuvre nomade recrutée chez les "barbares païens ", pour accomplir certains déplacements comme conducteurs de voitures de charge, gardiens de récolte ou guides de voyageurs. Sur le point de savoir qui étaient ces barbares, plusieurs interprétations sont possibles: ou bien, selon Ch. Courtois et J. Desanges, existaient vers 400 des Arzuges chrétiens ou païens de part et d'autre du limes; ou bien, pour Y. Modéran, (p. 458) une distinction serait à faire entre les Arzuges installés en territoire romain et les barbari qui seraient les tribus nomades venus d'audelà des frontières et qui ne cesseront d'exercer une pression de plus en plus forte sur les marges de l'Empire. Dans un tel contexte, on peut comprendre que le nom d'Arzugis ait fini par désigner cette entité frontalière sensible car vouée à la mobilité des hommes et qui correspond pour une large part au Nefzaoua actuel.

\section{BIBLIOGRAPHIE}

COURTOIS C., 1955 - Les Vandales et l'Afrique, Paris, p. 93-94.

DESANGES J., 1962 - Catalogue des tribus africaines de l'Antiquité classique à l'ouest du Nil, Dakar, p. 78-79.

DESANGES J., 1989 - « Arzuges », dans EB, VI, p. 950-952.

DESPOIS J. et RAYNAL R., 1967 - Géographie de l'Afrique du Nord-Ouest, Paris Payot, p. 428-430.

JANVIER Y., 1982 - La géographie d'Orose, Paris, Belles Lettres, p. 125-128. 
MANDOUZE A., 1982 - Prosopographie chrétienne du Bas-Empire, 1 Afrique (304-533), Paris, éd. CNRS, p. $522,621,1020$.

MODERAN Y., 2003 - Les Maures et l'Afrique romaine (IVe-VII ${ }^{e}$ siècle.), Rome, p. 364-373, 457-459.

MOREAU P., 1947 - Des lacs de sel aux chaos de sable, le pays des Nefzaouas, Tunis, Publications de l'Institut des Belles Lettres Arabes, $201 \mathrm{p}$.

MRABET A. et TROUSSET P., 2003 - « Axes de circulation, mobilité et contrôle des hommes dans la zone du limes d'Afrique ", dans Mobilité des Hommes et des Idées en Méditerranée, Actes du Colloque Sousse (mars 1999), éd. fac. des lettres et sciences humaines de Sousse, p. 65-88.

RACHET M., 1970 - Rome et les Berbères, Un problème militaire d'Auguste à Dioclétien, Bruxelles, Latomus, p. 163-164.

SETHOM H. et KASSAB A., 1981 - Les régions géographiques de la Tunisie, Tunis, p. 130-148.

TROUSSET P., 1982 - « Le franchissement des Chotts du sud tunisien dans l'Antiquité », Ant. afr., t. 18, p. $45-59$.

TROUSSET P., 2007 - « Une entité frontalière tardive et sa genèse ; des Nybgenii aux Arzuges ", article à paraitre dans Table Ronde Université de Caen.

INDEX

Mots-clés : Antiquité, Géographie, Tunisie 